\title{
HEAVY ELEMENT ABUNDANCES PREDICTED BY RADIATIVE SUPPORT THEORY IN THE ATMOSPHERES OF HOT WHITE DWARFS
}

\author{
P. Chayer, G. Fontaine, and F. Hesemael \\ Département de Physique, Université de Montréal
}

The surface composition of a white dwarf evolves as a result of the interaction of several mechanisms, the most important of which being gravitational settling. In the early phases of the evolution, theory shows that selective radiative levitation can occasionally defeat settling and, thus, prevent the formation of a pristine pure hydrogen (helium) atmospheric layer in a hot DA (non-DA) white dwarf (Fontaine and Michaud 1979; Vauclair, Vauclair, and Greenstein 1979). The exciting discovery of sharp metallic features in the ultraviolet spectra of several hot DA and non-DA stars alike resulting from the work of several investigators has provided the essential motivation for further theoretical investigations of radiative levitation in the atmospheres of white dwarfs. Additionnal impetus comes from the continuing investigations of hot DA white dwarfs carried out by Bruhweiler and Kondo wich have already revealed a most interesting observational pattern of heavy elements in these stars (Bruhweiler 1985). Moreover the recent awailability of theoretical equivalent widths of selected astrophysically important ultroviolet metal lines in hot DA white dwarfs (Henry, Shipman, and Wesemael 1985) makes a comparison between theory and observations -in at least this type of stars- a timely and useful exercise.

We have recently completed a detailed investigation of the effects of selective radiative support on $\mathrm{C}, \mathrm{N}, \mathrm{O}$, and $\mathrm{Si}$ in model atmospheres and envelopes of both $D A$ and non-DA white dwarfs. For each of these elements, and for a large number of models, we have derived the abundance profiles as a function of depth which are obtained by assuming an equilibrium between gravitational settling and radiative levitation. The bulk of our calculations are formally valid in optically thick layers only, i.e. in regions below the Rosseland photosphere. This is the same level of approximation which has been used so far in the few investigations devoted to radiative forces in white dwarfs (Vauclair, Vauclair, and Greenstein 1979; Morvan, Vauclair, and Vauclair 1986). Rodiative accelerations have also been computed for a small subset of models taking into account the effects of radiative transfer in optically thin layers and the non-uniform abundance profile on the synthetic spectra. As in the case of hot B subdwarfs discussed by Bergeron et a/. (1988), we find that the non-uniformity of the equilibrium abundance distribution of an element sup- 
ported by radiative levitation does not generally lead to line profiles and strengths which are markedly different from the line profiles and strengths obtained under the assumption of a uniform distribution with a value of the abundance equal to that of the non-uniform model at the Rosseland photosphere. The full results of our investigation will be presented elsewhere (Chayer et $\mathrm{a}$. 1988). In this short communication, we restrict ourselves to some sample results.

Fig.1 (Fig.2) shows the equilibrium abundance profiles for DA (non-DA) models with $M M_{\odot}=0.6$. Note that the envelope parameters have been taken from the evolutionary tracks of Winget, Lamb, and Van Hom (1988); the gravity therefore decreases (as it should) with increasing effective temperature. As compared to cosmic abundance ratios, only underabundances of $\mathrm{C}, \mathrm{N}, \mathrm{O}$, and $\mathrm{Si}$ can be supported by radiative levitation in the outermost layers of $D A$ white dwarfs with $M M_{\odot}=0.6$. The qualitative behavior of the equilibrium abundance profile is the same for $C, N$, and 0 . In particular, note that the surface abundance decreases monotonically with decreasing effective temperature for these elements. At high effective temperatures, there are two reservoirs supported by radiative forces. These two reservoirs are separated by a layer in which the dominant ionization state is that of the noble gas configuration which implies that radiative support is considerably reduced there. For $T_{e} \leqslant 40,000 \mathrm{~K}$, only the outer reservoir can be supported. The behavior of the surface abundance of $\mathrm{Si}$ is qualitatively different because that element goes through its noble gas configuration in the surface layers for the effective temperatures illustrated here. Thus, small traces of silicon can be supported at the photosphere of $0.6 M_{\odot} D A$ white dwarf models with $T_{e} \geqslant 75,000 \mathrm{~K}$, no support is possible in the range $75,000 \mathrm{~K} \geqslant T_{e} \geqslant 50,000 \mathrm{~K}$, and silicon can again reappear at the surfaces of cooler white dwarfs.

The surface abundances of the four elements considered all decrease monotonically with decreasing effective temperature in models of non-DA white dwarfs (Fig.2). At high effective temperatures $\left(T_{e} \geqslant 75,000 \mathrm{~K}\right)$, slight overabundances of $N$ can pollute the atmospheres of these stars, but, otherwise, underabundances are predicted. These are generally smaller than in the case of hot DA stars. However, the appearance of a superficial helium convective zone around $T_{e} \approx 65,000 \mathrm{~K}$ plays against radiative support in the cooler objects because radiative levitation can only be effective at the base of the convection zone where ionization is more complete and bound-bound absorption becomes less important. Below $\mathrm{T}_{\theta} \neq 35,000 \mathrm{~K}$, radiative support becomes totally negligible in the atmospheric layers of He-rich white dwarfs. The formation of a helium convection zone also prevents $\mathrm{Si}$ from reappearing at lower effective temperatures; no $S i$ is predicted in the atmospheres of $0.6 M_{\odot}$ non-DA white dwarfs with $T_{e} \leqslant 85,000 \mathrm{~K}$.

The results of our extensive calculations should be eventually used in a detailed comparison of the predictions of radiative support theory with the observations. Currently, such a comparison remains quite limited because detailed abundance analyses have been carried out for only three hot white dwarfs: the DA stars Wolf 
1346 and Feige 24 and the DO object PG 1034+001. Chayer et a/. (1987) have demonstrated that there are large discrepencies between the predicted and observed $C$ abundances for both Feige 24 and PG 1034+001. They suggest that another mechanism (such as a weak wind) possibly interferes with settling and radiative levitation in these hot objects. At the very least, it appears that the idea of a simple equilibrium between gravitationel settling and radiative support does not pass the test in these stars.

Further evidence in that direction is gained by using the sample of some 20 bright, hot DA white dwarfs currently being investigated by Bruhweiler and Kondo. Pending the final results of their detailed studies, Bruhweiler (1985) has nevertheless announced a most interesting observational pattern of heavy elements in hot DA stars: while no features are detected (at the $15 \mathrm{~mA}$ level) in high-resolution /LE spectra of a minority of objects, only Si features are observed for stars with $\mathrm{T}_{e}\{40,000 \mathrm{~K}$ in the rest of the sample, and features of $\mathrm{Si}, \mathrm{C}$, and $\mathrm{N}$ are observed in the hotter stars. This qualitative pattern can be contrasted to the predictions of radiative support theory. As an illustrative example, we have used the predicted abundances at the Rosseland photosphere of our $0.6 M_{\odot} D A$ models to derive the strenghts of several spectral features which are accessible in the llE window. In this process, we have used the equivalent widths computed in the spectrum synthesis study of Henry, Shipman, and Hesemael (1985). The results are shown in Fig.3 which puts in evidence the predicted equivalent width of a given spectral line as a function of effective temperature. Oxygen features have not been considered by Henry, Shipman, and Wesemael (1985) because they are only of relevance to effective temperatures higher than that of the hottest known DA white dwarf in the IUF spectral range. Even adopting a very conservative detection limit of $100 \mathrm{~mA}$, we find that the observed abundance pattern is not reproduced as carbon features for example would still be easily detectable in DA white dwarfs with $T_{e} \leqslant 40,000 \mathrm{~K}$. Coupled to the fact that no features are observed in some of the DA objects in the Bruhweiler and Kondo sample, this suggests very strongly that the predictions of simple radiative support theory are inadequate. He note that the absence of metallic spectral features in some of the hat DA stars observed with the IUE in the high resolution mode is usually blamed on known (or expected) higher gravities in these stars. Preliminary inspection of our detailed results indicates that, while the expected photospheric abundances are indeed reduced in higher gravity objects, spectral features should still be detectable.

The main conclusion of this paper is that simple radiative support theory fails to explain the observed abundance pottem of heavy elements in hot white dwarfs. At least one other mechanism must interfere with gravitational settling and radiative levitation in such objects. We note that the studies of Bruhweiler and Kondo (1981, 1983) already point to a very natural candidate: the presence of weak winds. The work of these investigators strongly suggests that the outermost layers of very hot white dwarfs are permeated with a wind which eventually dies out with cooling. It is important to point out that such winds do not need to, and indeed cannot, be 
very large. This is because abundance anomalies (the large underabundances usually observed in white dwarfs) connot be built if the wind velocity is much larger than the diffusion velocities of various species. Michaud (1987) finds somewhat weak constraints of $3 \times 10^{-11}-3 \times 10^{-16} \mathrm{M}_{\odot} \mathrm{yr}^{-1}$ for the maximun mass loss rate allowing chemical sedimentation and settling in white dwarfs. He note that a mass loss rate as small as $10^{-21} \mathrm{M}_{\Theta} y r^{-1}$ can still play havoc with the predicted surface abundances in presence of radiative support. For example, we connot help but notice that the outer reservoirs of $C$ and $N$ are opened at the surface of a DA white dwarf and could easily be emptied by such $a$ wind in the hot phases of the evolution (Fig.1). At the same time the reservoir of $S i$ is not opened at the surface in a significant effective temperature range. Hence, it is strongly tempting to speculate that first $C$ and then $N$ are totally expelled from the photosphere of DA white dwarts in the very hot phases of the evolution, while Si "hides" below the surface until it is caught by the effects of wind and becomes eventually visible in the later phases of the evolution. A dependance on gravity for the effects of winds is also easily envisioned. Whether or not this speculative scenario is at all correct rests with a detailed investigation of the interaction between settling, radiative levitation, and mass loss in hot white dwarts. We have embarked on such a project and will report our results in due time.

This work was supported by NSERC Canada, and by a E.WR. Steacie Fellowship to one of us (GF).

Bergeron, P., Wesemael, F., Michaud, G., and Fontoine, G. 1988, Ap. J., in press. Bruhweiler, F.C. 1985, Bull. Aner. Astron. Soc., 17, 559.

Bruhweiler, F.C., and Kondo, Y. 1981, Ao. J. (Letters), 248, L123.

Bruhweiler, F.C., and Kondo, Y. 1983, Ap. J., 269, 657.

Chayer, P., Fontaine, G., Hesemael, F. and Michaud, G. 1987, in IAU Colloquium No. 95, The Second Conference on Faint Blue Stars,

A. G. D. Philip, D. S. Hayes and J. Liebert, eds., L. Davis Press,

Schenectady, P. 653.

Chayer, P., Fontaine, G., Hesemael, F. and Michaud, G. 1988, in preparation. Fontaine, G., and Michoud, G. 1979, Ap. J., 231, 826.

Henry, R.B.C., Shipman, HL., and Wesemael, F. 1985, Ap. J. Supp/., 57, 145.

Michaud, G. 1987, in IAU Colloquium No. 95. The Second Conference on

Faint B/we Sters, A. G. D. Philip, D. S. Hayes and J. Liebert, eds., L. Davis Press, Schenectady, p. 249.

Moruon, E., Vauclair, G., and Vauclair, S. 1986, Astr. Ap., 163, 145.

Vauclair, G., Vauclair, S., and Greenstein, JL. 1979, Astr. Ap., 80, 79.

Winget, DE., Lamb, D.Q., and Van Horn, HM. 1988, in preparation. 

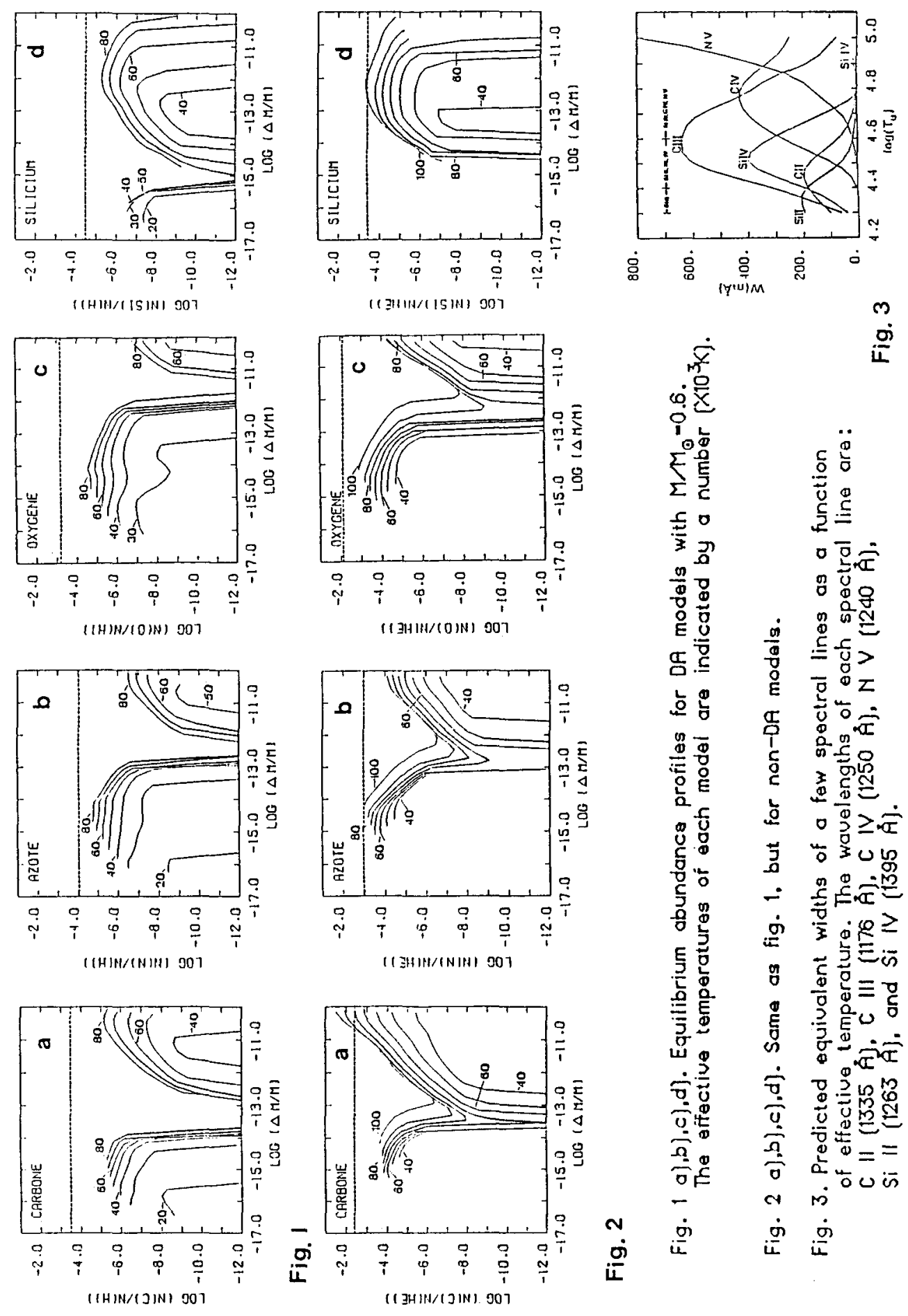